\title{
CLASS NUMBERS OF PURE FIELDS
}

\author{
R. A. MOLLIN
}

\begin{abstract}
Necessary and sufficient conditions are given for the class number $h_{K_{i}}$ of a pure field $K=Q\left(m^{1 / p^{i}}\right)$ (for $\left.i=1,2\right)$ to be divisible by $p^{r}$ for a given positive integer $r$ and prime $p$. Moreover the divisibility of $h_{K_{i}}$ by $p$ is linked with the $p$-rank of the class group of the $K(\varsigma)$ and prime divisors of $m$, where $\zeta$ is a primitive $p$ th root of unity.

Finally we prove in an easy fashion that for a given odd prime $p$ and any natural number $t$ there exist infinitely many non-Galois algebraic number fields (in fact pure fields) of degree $p^{i}(i=1,2)$ over $Q$ whose class numbers are all divisible by $p^{t}$.
\end{abstract}

1. Introduction. Pure cubic, quartic, quintic, and sextic fields have been extensively studied by many authors (for example see [3-4] and [8-12]). Parry and Walter [11] studied the Galois closure $L=Q(\zeta, \sqrt[p]{m})$ of pure fields $K_{1}=Q(\sqrt[p]{m})$ of prime degree and classified those $m$ for which the class number $h_{L}$ of $L$ is relatively prime to $p$. However necessary and sufficient conditions (for arbitrary $p$ ) such that $h_{K}$ is divisible by $p$ have failed to make their way into the literature. Our first result is to give such conditions for regular primes. We use this result as a tool for linking the divisibility of $h_{K}$ by $p$ with the rank of the Sylow $p$-subgroup of the class group of $L$ and also with certain primes dividing $m$. Moreover for the pure fields $K_{2}=Q\left(\sqrt[p^{2}]{m}\right)$ of prime squared degree we obtain necessary conditions and sufficient conditions for $h_{K_{2}}$ to be divisible by $p$, and use this result as a tool to provide applications similar to that of $K_{1}$ described above.

Finally when $m$ is divisible by $t$ primes congruent to 1 modulo $p$ we give an explicit description of an unramified extension of $K_{i}(i=1$ or 2$)$ of degree $p^{t}$ (therefore of infinitely many such $K_{i}$ ).

2. Pure fields. Throughout the remainder of the discussion the following notation will be in force.

$Z=$ the ring of rational integers.

$Q=$ the field of rational numbers.

$p=$ an odd rational prime.

$m>1$, a $p$-power free rational integer.

$\varsigma=$ a primitive $p$ th root of unity.

$k=Q(\zeta)=$ the $p$ th cyclotomic field.

$K_{i}=Q\left(m^{1 / p^{i}}\right)$, a pure field of degree $p^{i}$, where $i=1$ or 2 .

$L_{i}=K_{i} k$, where $i=1$ or 2 .

$G\left(F_{1} / F_{2}\right)=$ the Galois group of a normal extension $F_{1} / F_{2}$ of number fields.

Received by the editors January 31, 1985.

1980 Mathematics Subject Classification (1985 Revision). Primary 12A50; Secondary 12A40, A2A55.

The author's research is supported by N.S.E.R.C. Canada, grant number A8484. 
$U(F)=$ the group of units of the ring of integers of a number field $F$.

$h_{F}=$ the class number of a field $F$.

$C_{F}=$ the ideal class group of a number field $F$.

$G(p)=$ the Sylow $p$-subgroup of a group $G$.

$r(F, p)=$ the rank of $C_{F}(p)$.

$|y|_{p}=$ the $p$-primary part of $y \in Z$.

$a_{1}(p)=1+(p-3)^{2} / 4$.

$a_{2}(p)=(p-1)^{3} / 4$.

$b_{1}(p)=\left(p^{2}-5\right) / 4$.

$b_{2}(p)=(p-1)^{3} / 4-(p-3) / 2$.

$Q_{1}=\left|U\left(L_{1}\right): U(k) \prod_{i} U\left(K_{1}\right)_{i}\right|$ with the product ranging over the conjugates $\left(K_{1}\right)_{i}$ of $K_{1}$ over $Q$.

$Q_{2}=\left|U\left(L_{2}\right): U\left(L_{1}\right) \prod_{i} U\left(K_{2}\right)_{i}\right|$ with the product ranging over the conjugates $\left(K_{2}\right)_{i}$ of $K_{2}$ over $K_{1}$.

Finally we assume throughout that $p$ is regular, i.e. that $p$ does not divide $h_{k}$.

Now, we begin with a result which includes necessary conditions and sufficient conditions (but unfortunately not necessary and sufficient) for $\left.h_{K_{i}}\right|_{p}=p^{r}$ for a given positive integer $r$. This result was motivated by the quintic $(p=5, r=1)$ case given by Parry [8] of which part (i) of the following may be considered to be a generalization, and part (ii) provides a generalization of the cubic case $(p=3, r=1)$ provided by Walter [12], which motivated this second result.

THEOREM 2.1. Let $r$ be a positive integer.

(i) If $p^{a_{1}(p)+(r-1)(p-1)}$ divides $h_{L_{1}}$, then $p^{r}$ divides $h_{K_{1}}$. Conversely if $p^{r}$ divides $h_{K_{1}}$ and $p^{(p-2)(p-3) / 2}$ divides $Q_{1}$, then $p^{a_{1}(p)+(r-1)(p-1)}$ divides $h_{L_{1}}$. Furthermore, if $p$ divides $h_{K_{1}}$, then $p$ divides $h_{L_{1}}$.

(ii) Assume that $p$ does not divide $h_{L_{1}}$. Then $p^{a_{2}(p)+(r-1)(p-1)}$ divides $h_{L_{2}}$ implies that $p^{r}$ divides $h_{K_{2}}$. If $p>3, p^{r}$ divides $h_{K_{2}}$, and $p^{(p-4)(p+1) / 2}$ divides $Q_{2}$, then $p^{a_{2}(p)+(r-1)(p-1)}$ divides $h_{L_{2}}$. Finally, if $p$ divides $h_{K_{2}}$, then $p$ divides $h_{L_{2}}$.

PROOF. (i) We first note the following formulas obtained from Walter [12]:

(2.2) $h_{L_{1}} p^{b_{1}(p)}=Q_{1} h_{k} h_{K_{1}}^{p-1}$, and

(2.3) $Q_{1}$ divides $p^{(p-1)(p-2) / 2}$.

Now we assume that $p^{a_{1}(p)+(r-1)(p-1)}$ divides $h_{L_{1}}$. Hence from (2.2) we have that $\left|h_{k}\right|_{p}^{1-p} p^{a_{1}(p)+b_{1}(p)+(r-1)(p-1)}$ divides $Q_{1}$. But

$$
a_{1}(p)+b_{1}(p)=1+(p-1)(p-2) / 2 .
$$

Thus we get that $p^{r}$ divides $h_{K_{1}}$ from (2.3).

Conversely from (2.2) we have that $p^{((p-2)(p-3) / 2)+r(p-1)-b_{1}(p)}$ divides $h_{L_{1}}$. But $(p-2)(p-3) / 2=a_{1}(p)+b_{1}(p)-(p-1)$. Hence $p^{a_{1}(p)+(r-1)(p-1)}$ divides $h_{L_{1}}$. The last statement of part (i) follows from Iwasawa [6], since there is a $K_{1}$-prime above $p$ which is totally ramified in $L_{1}$.

(ii) From Walter [12] we have

(2.4) $p^{b_{2}(p)} h_{L_{2}} h_{K_{1}}^{p-1}=Q_{2} h_{L_{1}} h_{K_{2}}^{p-1}$, and

(2.5) $Q_{2}$ divides $p^{p(p-1)(p-2) / 2}$.

We first assume that $p^{a_{2}(p)+(r-1)(p-1)}$ divides $h_{L_{2}}$. Then from (2.4) we have that $\left|h_{K_{2}}\right|_{p}^{1-p} p^{a_{2}(p)+b_{2}(p)+(r-1)(p-1)}$ divides $Q_{2}$. But $a_{2}(p)+b_{2}(p)=1+p(p-1)(p-2) / 2$. Thus (2.5) yields that $p^{r}$ divides $h_{K_{2}}$. 
Conversely if $p^{r}$ divides $h_{K_{2}}$, then from (2.4) we have that $h_{L_{2}}$ is divisible by $p^{((p-4)(p+1) / 2)+r(p-1)-b_{2}(p)}$. But $(p-4)(p+1) / 2=a_{2}(p)+b_{2}(p)-(p-1)$. Hence $p^{a_{2}(p)+(r-1)(p-1)}$ divides $h_{L_{2}}$.

Finally the last statement of the theorem is immediate from Iwasawa [6]. Q.E.D.

We note that the above conditions are the "best possible", in the sense of being minimal. This fact is illustrated by the simplest case where $r=1$ and $p=3$, wherein we have $a_{1}(p)+(r-1)(p-1)=1$. We have from Theorem 2.1 that if 3 does not divide $h_{L_{1}}$, then 3 does not divide $h_{K_{1}}$. Conversely if 3 does not divide $h_{K_{1}}$, then, since $Q_{1}=3$, we have that 3 does not divide $h_{L_{1}}$ from (2.2), i.e. for $p=3$ we have $p \mid h_{K_{1}}$ if and only if $p \mid h_{L_{1}}$. Moreover the necessary and sufficient conditions for $p$ to divide $h_{L_{1}}$ were given by Parry and Walter [11]. Finally, in this connection we note that it is not enough to know $p$-divisibility conditions for $h_{L_{1}}$ in order to settle the question for $h_{K_{1}}$. We see this already for $p=5$. Since $5^{3} \mid Q_{1}$ (see Parry [8]), then $5 \mid h_{K_{1}}$ if and only if $5^{2} \mid h_{L_{1}}$ from Theorem 2.1.

The following result links the rank of $C_{L_{i}}(p)$ to the divisibility of $h_{K_{i}}$ by $p$.

THEOREM 2.2. Suppose that $p^{c}$ divides $Q_{i}$, where $c=(p-2)(p-3) / 2$ if $i=1$ and $c=(p-4)(p+1) 2$ if $i=2$. Then if $r\left(L_{i}, p\right)<p-1$ and either $p>7$ or $i=2$ then $p$ does not divide $h_{K_{i}}$. If $i=1,3<p \leq 7$ and $r\left(L_{1}, p\right)=1$, then $p$ does not divide $h_{K_{1}}$.

ProOF. By Theorem 2.1, if $p$ divides $h_{K_{i}}$, then $p^{a_{i}(p)}$ divides $h_{L_{i}}$. Now if $C_{L_{i}}(p)$ has an element of order $p^{2}$, then by Cornell and Rosen [2, Theorem 5, p. 7] we have that $r\left(L_{i}, p\right) \geq p-1$. Thus $C_{L_{i}}(p)$ must be elementary abelian which implies that $r\left(L_{i}, p\right) \geq a_{i}(p)$. Hence $p-1 \geq a_{i}(p)$ if $p>7$ or $p=2$, a contradiction. If $3<p \leq 7$ and $i=1$, then $1 \geq a_{1}(p)$, again a contradiction. Q.E.D.

We isolate the following special case which motivated the above.

COROLLARY 2.1 (PARRY [8]). If $p=5$ and $5 \mid h_{K_{1}}$, then $C_{L_{1}}(p)$ is not cyclic.

The final result actually gives an explicit description of an unramified extension $F$ of $K_{i}$ of degree $p^{t}$ whenever $m$ is divisible by $t$ primes $q \equiv 1(\bmod p)$. The following is a generalization of the cubic case by Honda [3] which motivated our result. It is also a generalization of the quintic case by Parry [8]. In what follows $\zeta_{q}$ denotes a primitive $q$ th root of unity.

Proposition 2.1. Suppose that $m$ is divisible by $t \geq 1$ primes $q \equiv 1$ (mod $p)$. Let $F^{(q)}$ be the subfield of $Q\left(\zeta_{q}\right)$ such that $\left|F^{(q)}: Q\right|=p$, and let $M$ be the compositum of the $t F^{(q)}$ 's. Then $M K_{i}$ is unramified over $K_{i}$, i.e. $p^{t}$ divides $h_{K_{i}}$.

PROOF. It is a straightforward application of Abhyankar's lemma (e.g. see [1, Theorem 3, p. 504] that $M K_{i}$ is unramified over $K_{i}$. Q.E.D.

Note that in the above result we did not require that $p$ be regular. Therefore we have the following proposition as an immediate consequence.

Proposition 2.2. Let $p$ be an odd prime. Then given any natural number $t$ there exist infinitely many non-Galois algebraic number fields of degree $p^{i}(i=$ 1 or 2) over $Q$, whose class numbers are all divisible by $p^{t}$.

Note that the above is a generalization of the main result of Ishida [5, Theorem 1 , p. 65]. Moreover our proof is much easier than that given in [5]. 


\section{REFERENCES}

1. G. Cornell, On the construction of the relative genus field, Trans. Amer. Math. Soc. 271 (1982), 501-511.

2. G. Cornell and M. Rosen, Group theoretic constraints in the structure of the class group, J. Number Theory 13 (1981), 1-11.

3. T. Honda, Pure cubic fields whose class numbers are multiples of three, J. Number Theory 3 (1971), 7-12.

4. K. Iimura, A criterion for the class number of a pure quintic field to be divisible by 5 , J. Reine Angew. Math. 292 (1976), 201-210.

5. M. Ishida, A note on class numbers of algebraic number fields, J. Number Theory 1 (1969), 65-69.

6. K. Iwasawa, A note on class numbers of algebraic number fields, Abh. Math. Sem. Univ. Hamburg 20 (1956), 257-258.

7. R. Mollin, Class numbers and a generalized Fermat theorem, J. Number Theory 16 (1983), 420429.

8. C. J. Parry Class number relations in pure quintic fields, Symposia Math. 15 (1975), 475-485.

9. __ Class number relations in pure sextic fields, J. Reine Angew. Math. 274/275 (1975), 360375.

10. 102-112.

11. C. J. Parry and C. D. Walter, The class number of pure fields of prime degree, Mathematika 23 (1976), 220-226.

12. C. D. Walter, Pure fields of degree 9 with class number prime to 3, Ann. Inst. Fourier Grenoble 30 (1980), 1-15.

Department of Mathematics, University of Calgary, Calgary, Alberta, T2N 1N4, CANADA 